\title{
Ear Involvement in Inflammatory Bowel Disease: A Review of the Literature
}

\author{
Fotios S. Fousekis ${ }^{\mathrm{a}}$, Maria Saridi ${ }^{\mathrm{b}, \mathrm{c}, \mathrm{g}}$, Eleni Albani ${ }^{\mathrm{d}}$, Fady Daniele ${ }^{\mathrm{e}}$, Konstantinos H. Katsanos ${ }^{\mathrm{a}}$, \\ Ioannis G. Kastanioudakis ${ }^{\mathrm{f}}$, Dimitrios K. Christodoulou ${ }^{\mathrm{a}}$
}

\begin{abstract}
Inflammatory bowel disease (IBD) is a multisystemic disease. The ear is a rare but recognized site of extraintestinal manifestations of IBD. In external ear, the more common manifestations of IBD are pyoderma gangrenosum, metastatic Crohn's disease and relapsing polychondritis and the treatment includes corticosteroids and antiTNF agents. Sensorineural hearing loss (SNHL) is the most common ear disease in IBD and especially in patients with ulcerative colitis. In most cases of IBD patients with SNHL, the hearing loss is attributable to autoimmune inner ear disease (AIED). Diagnosis of AIED is based on clinical presentation, the demonstration of a progressive sensorineural hearing loss in periodic audiological tests, a response to immunosuppressive drugs and exclusion of other causes of SNHL. The only diagnostic test that is available for clinical use is the Otoblot test (Western blot for antibodies against $68 \mathrm{kD}$ protein-inner ear antigens). Initial therapy is usually steroids, with a step up to antiTNF-a therapy and cochlear implantations with failure of treatment. Furthermore, Cogan's syndrome, a chronic disease characterized by deafness, vertigo keratitis and aortitis, has been associated with IBD and mainly with Crohn's disease.
\end{abstract}

Keywords: Crohn's disease; Ulcerative colitis; Autoimmune inner ear disease; Sensorineural hearing loss; Extraintestinal manifestations

\section{Introduction}

Inflammatory bowel disease (IBD) is an inflammatory disor-

Manuscript submitted May 6, 2018, accepted May 15, 2018

a Department of Gastroenterology, Medical School of Ioannina, Ioannina, Greece

bDepartment of Social and Educational Policy, University of Peloponnese, Corinth, Greece

'Hellenic Open University, Patras, Greece

dNursing Department, Technological University of Patras, Patra, Greece 'Melbourne Medical School, The University of Melbourne, Melbourne, Australia

fDepartment of Otolaryngology, Medical School of Ioannina, Greece

g Corresponding Author: Maria Saridi, General Hospital of Korinthos, Sina 33

Street, Corinth, Greece. Email: sarmar32@windowslive.com

doi: https://doi.org/10.14740/jocmr3465w der that affects the gastrointestinal tract. However, IBD is a systemic disorder with various extraintestinal manifestations, which may include the ear. The ear has been associated with a wide variety of other systemic autoimmune disorders such as systemic lupus erythematosus, rheumatoid arthritis, Behcet's disease and Sjogren's syndrome. Three possible mechanisms for autoimmune related otologic disorders have been suggested: 1) autoantibody binding to type II collagen or other otologic components (type II immunologic injury); 2) immune complex formation leading to vasculitis (type III); 3) T cellmediated auto reactivity to inner ear membranous elements (type IV) [1]. There is evidence to support external, middle and inner ear involvement in IBD. In this systematic review, we present the various types of otologic disorders that are correlated with IBD, and the therapy and management of these diseases.

\section{External and Middle Ear Involved in IBD}

External ear involvement in IBD is very rare. Usually, external ear involvement is due to concurrent diseases such as pyoderma gangrenosum (PG), metastatic Crohn's disease, necrotizing external otitis and relapsing polychondritis.

PG is a serious ulcerating skin disease and often coexists with systemic disease, with rheumatoid arthritis and IBD being the most common $[2,3]$. While PG is more commonly found in the lower limbs, $25 \%$ of patients with PG have confirmed lesions on the head and neck. However, lesions on auricular areas are still very rare. There have been reported cases of patients with IBD and auricular PG causing tissue necrosis and ear swelling. Biopsy of the affected area greatly helps in diagnosis in these patients $[4,5]$. The first-line treatment for PG is oral corticosteroids. If patients do not respond, anti-TNF-a factors are the second-line treatment [6] as it seems that infliximab and adalimumab are effective and safe in patients with IBD and PG [7]. There has been a case reported of a woman with a history of ulcerative colitis and primary sclerosing cholangitis who developed PG on the right ear. Treatment with cyclosporine $(10 \mathrm{mg} / \mathrm{kg})$ was successfully administered, which also induced remission of the patient's cholangitis and ulcerative colitis [8].

Metastatic Crohn's disease is a cutaneous granulomatous non-caseating lesion that occurs in patients with $\mathrm{CD}$. The clinical presentation and microscopic findings are necessary for accurate diagnosis. Two cases of metastatic $\mathrm{CD}$ with involve- 
ment of the retro-auricular area have been described $[9,10]$. In one case, a patient with perianal $\mathrm{CD}$ received oral steroids and infliximab and the therapy resulted in rapid remission of the cutaneous auricular lesion and Crohn's disease [9]. In the other case, a 10-year-old boy presented with bilateral chronic granulomatous external otitis and obliteration of the external auditory canal. Histopathologic findings suggested cutaneous $\mathrm{CD}$ and a subsequent gastroenterologic workup confirmed the diagnosis of CD [11].

Relapsing polychondritis (RP) is a rare multisystemic inflammatory disease of unknown etiology, which involves cartilaginous structures, predominantly those of the ears, nose and upper and lower respiratory tracts. It believed that disease has an autoimmune etiology due to presence of anticollagen type II and anti-matrilin-1 antibodies, infiltrating T cells and the observation that the immunosuppressive agents suppress the disease [12]. Relapsing polychondritis has been associated with other autoimmune diseases in approximately one third of patients, including rheumatoid arthritis, IBD, Behcet's syndrome, systemic lupus erythematosus, and other vasculitis and hematological disorders [13]. Auricular chondritis occurs in the majority of patients with relapsing polychondritis causing unilateral or bilateral swelling, auricular pain and redness. Diagnosis is made clinically and the McAdam criteria can be helpful in guiding this [14]. Three cases of IBD patients with auricular relapsing polychondritis have been reported. In one case the patient had Crohn's disease [15] and in the other two cases the patients were suffering from UC $[16,17]$. The treatment of RP includes corticosteroids, immunodulators and biologic agents. The goal of treatment is to abate the symptoms and to preserve the integrity of cartilaginous structures.

While immunosuppressive therapy is necessary for the remission and maintenance of IBD, this increases the risk of infectious complications. Duque et al described a 27-year-old female with $\mathrm{CD}$ treated with azathioprine and infliximab. She complained of left otalgia, otorrhea and fever with no gastrointestinal complaints. She suffered from necrotizing external otitis and she was successfully treated with ciprofloxacin (500 $\mathrm{mg}$ IV for 3 weeks and then oral for another 3 weeks), ceftazidime (1,000 mg IV for 6 weeks), as well as topical therapy with alcohol and aspiration of secretions. Necrotizing external otitis is a rare but acknowledged complication of external otitis and affects predominantly immunocompromised patients and diabetics [18].

Data are limited with regard to diseases of the middle ear and its association with IBD. Shaw SY et al compared 294 children with IBD with 2,377 controls based on age, sex and geographical region. They studied the association between early childhood otitis media and pediatric inflammatory bowel disease. The study showed that children with an otitis media diagnosis by 5 years have 2.8 -fold $(95 \%$ CI, $1.5-5.2 ; \mathrm{P}=$ 0.001 ) higher risk for IBD development [19]. A possible explanation is that the children with otitis media received antibiotics and the antibiotic use is a possible predictive factor in IBD, as a study showed that patients with IBD are more likely to have been prescribed antibiotics $2-5$ years before their diagnosis [20].

\section{Inner Ear Involved in IBD}

\author{
Sensorineural hearing loss (SNHL)
}

SNHL has been reported as an extraintestinal manifestation of IBD and especially in patients with UC. SNHL is the most common ear disease in patients with IBD. In a retrospective observational study with 32 IBD patients with otologic problems, SNHL was found in 22 of 32 patients. Nineteen of 22 patients had no identifiable causes for SNHL. Only one patient responded to medical treatment [21]. Also, it seems that subclinical SNHL is common in IBD patients. The presence of subclinical SNHL was investigated in 39 patients with IBD (18/UC, 21/CD) and 25 healthy controls and the findings between two groups were compared. A significant difference was found at high frequencies in UC patients, at frequencies of 2, 4 and $8 \mathrm{kHz}$ and in $\mathrm{CD}$ patients at the frequency of $4 \mathrm{kHz}$ [22]. However, there was no difference when Kalyoncu et al performed otoscopy, tympanometry and pure tone tympanometry in 24 pediatic patients with IBD and 20 healthy children and compared the findings between these groups [23].

\section{Autoimmune inner ear disease (AIED)}

In most cases of IBD patients with SNHL, the hearing loss is attributable to autoimmune inner ear disease. AIED is an idiopathic inflammatory disease of inner ear characterized by rapidly progressive bilateral SNHL. The hearing level often fluctuates, with period of deterioration alternating with remission. About $50 \%$ of patients with AIED also have vestibular symptoms in addition to hearing loss. AIED can be present alone (primary AIED) or associated with other autoimmune disease such as rheumatoid arthritis, systemic lupus erythematosus and IBD, mainly ulcerative colitis (secondary AIED). Diagnosis of AIED is based on clinical presentation, the demonstration of a progressive sensorineural hearing loss in periodic audiological tests, a response to immunosuppressive drugs such as corticosteroids and exclusion of other causes of SNHL [24].

The response to immunosuppressive therapy has led to an investigation to identify autoantibodies to inner ear components. Harris and Sharp used bovine inner ear extract in Western blot assays and detected antibody to a $68-\mathrm{kD}$ inner ear antigen in 19 of 54 patients (35\%) with progressive SNHL [25]. H68-kD protein is an antibody to Heat Shock Protein 70 (HSP70). Furthermore, a study confirmed the finding of circulating antibodies against a $68-\mathrm{kD}$ protein that was detected in the serum of 42 out of 72 patients with idiopathic progressive bilateral SNHL. Patients who were antibody-positive compared with antibody-negative responded to steroid treatment more frequently ( $75 \%$ vs. $18 \%, \mathrm{P}=0.001$ ) [26]. For clinical use is available the Otoblot test, which is a Western blot for antibodies against a $68 \mathrm{kD}$ protein found in the inner ear. However in a study demonstrated, that only $9 \%$ of patients with AIED were HSP70-positive [27]. Moreover in another study, the frequency of antibodies to HSP substrates did not differ 


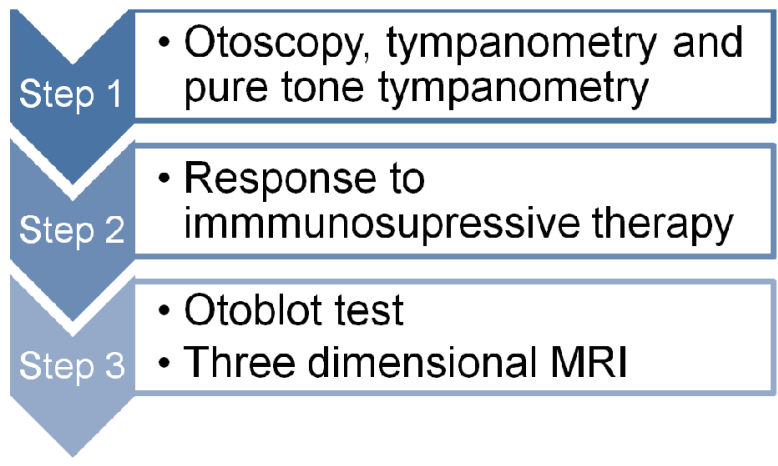

Figure 1. Diagnostic tests for sensorineural hearing loss in patients with IBD.

in patients with AIED and controls [28]. Currently, the use of Otoblot test in the clinical practice is controversial [29].

Diagnostic tests for SNHL in patients with IBD include step by step: 1) Otoscopy, tympanometry and pure tone tympanometry; 2) Response to immmunosupressive therapy and 3) Otoblot test and three dimensional MRI. There is minimal data on the role of imaging in the diagnosis and management of AIED. In two cases of ulcerative colitis and severe SNHL, three-dimensional magnetic resonance imaging revealed obliteration of the inner ear. These patients responded poorly to steroid therapy [30]. Thus, the Otoblot test and three-dimensional MRI appear to play a limited diagnostic role in IBD patients with SNHL and possibly AIED (Fig. 1).

The therapy of AIED includes immunosuppressive agents, largely corticosteroids. Overall steroids response rates are approximately $70 \%$ [31]. Also, it seems that cytokines play a significant role in pathogenesis of disease and response of treatment, as TNF expression has correlated with steroid-sensitive AIED [32] and expression of interleukin-1 has associated with steroid resistant AIED [33]. Furthermore, a study showed that the level of TNF can be used as both as a diagnostic and prognostic factor for AIED. They studied the level of TNF in 85 patients with clinical and audiometric characteristics of AIED as well as 11 control subjects and found that patients with suspected AIED had a higher level of TNF compared with healthy subjects, and that TNF levels were higher in patients who were nonresponsive to steroids than those who were responsive to steroids [32]. Therefore, the use of anti-TNF therapy may be an alternative treatment in patients with no response to steroids, but clinical data is still unclear. In a retrospective study, eight patients with AIED who had previously not responded to steroid therapy and/or treatment with other immunosuppressive agents received infliximab. After the completion of treatment, no patient had a positive response to infliximab therapy by objective measurements, and only one patient reported subjective improvement [34]. Heywood et al described a case of non responsive AIED that was improved by infliximab; the disease relapsed after discontinuation of infliximab and was again improved after reintroduction [35]. In another case, a patient with Crohn's disease and AIED received infliximab. Anti-TNF-a therapy resulted in improvement in hearing of 15 $\mathrm{dB}$ on average across all frequencies. Hearing remained stable afterwards [36].

In some cases, there is no response to pharmacological therapy and therefore undergo cochlear implantation. It has been described a patient with diagnosed CD and bilateral SNHL as a result of possible AIED. The patient was treated with immunosuppressive therapy during the development of SNHL. Treatment of SNHL included corticosteroids, azathioprine and anti-TNF agents. There was a lack of response, and the patient subsequently lost his hearing and underwent bilateral cochlear implantation [37].

Therefore, currently there is a step-up therapy for AIED. First-line treatment is steroid use and second line treatment is the anti-TNF agents. Third line treatment is the cochlear implantation, if the medication fails (Fig. 2).

A correlation between IBD and AIED has been shown, even in pediatric IBD patients. Kumar et al described a case in a 12-year-old boy with UC and AIED which initially responded to steroid therapy, but who progressed 4 years later resulting in profound bilateral hearing loss in spite of good control of IBD [38]. While there have been many cases of remission of autoimmune diseases during pregnancy, it seems that pregnancy does not affect the course of AIED in IBD patients. A case report describes the case of a 31-year old woman with diagnosed $\mathrm{CD}$ who presented with acute total deafness of one ear and SNHL in the other ear, both of which occurred after she got pregnant. She responded to steroid treatment and her hearing improved [39].

\section{Melkersson-Rosenthal syndrome}

Melkersson-Rosenthal syndrome is a rare clinical entity characterized by the following triad: facial nerve palsy, swelling of lips and a fissured tongue. However, otologic symptoms can coexist with the classic symptoms of the syndrome. This syndrome is strongly associated with CD and is usually a mani-

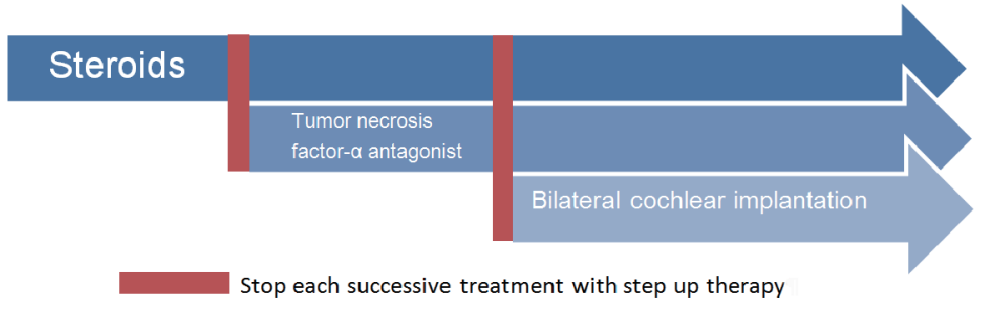

Figure 2. Step up treatment of autoimmune inner ear disease. 
festation of $\mathrm{CD}$ [40]. In one case that described a 30-year-old woman with $\mathrm{CD}$ and Melkersson-Rosenthal syndrome, who developed severe ear pain as part of the clinical course [41].

\section{Cogan's syndrome}

Cogan's syndrome is a rare chronic autoimmune inflammatory disease, characterized by bilateral sensorineural hearing loss, vestibular symptoms, non-syphilitic interstitial keratitis and aortitis. In many patients, Cogan's syndrome is related with other autoimmune disease such as rheumatoid arthritis, Sjogren's syndrome and IBD. A retrospective observational study was done with 22 concomitant Cogan's syndrome-IBD cases, with six patients with underlying UC and 16 with $\mathrm{CD}$. IBD was diagnosed before the onset of Cogan's syndrome in all of these patients, with a mean duration of IBD of 8.7 years. Also, IBD activity did not seem to trigger Cogan's syndrome, as 11 of 22 patients had inactive disease at the time of Cogan's syndrome diagnosis. Immunosuppressive treatment does not seem to prevent the onset of disease, as sixteen patients received immunosuppressive agents, of whom six received anti TNF-a therapy [42]. Corticosteroids are often effective in controlling the disease. If the patient does not improve, immunosuppressive drugs, such as methotrexate and azathioprine, are used. Infliximab is a promising treatment option, enabling steroid tapering and leading to improvement in auditory/ocular disease, with better outcomes if administered in the early stages of the disease [43]. Therefore, Cogan's syndrome could be another indication for use of anti-TNF-a agents in patients with IBD.

\section{Drug-induced ototoxicity and IBD}

There have been no reports of IBD patients with drug-induced ototoxicity. The most ototoxic drugs in IBD therapy are 5-ASA analogues, ciprofloxacin and metronidazole. 5-ASAs are used often for maintenance and remission in IBD. While salicylate overdose can cause reversible SNHL and tinnitus [44], so far no IBD patient treated with 5-ASA had otic side effects. Metronidazole and ciprofloxacin are often used in fistulizing Crohn's disease therapy. There have been few cases of SNHL due to oral administration of metronidazole. The hearing loss recovered after drug withdrawal and oral steroids in these cases [45]. Ciprofloxacin has been associated with several cases of tinnitus, which generally ends when the medication is ceased [46].

\section{Conclusions}

IBD can be associated with ear disease in all components of the ear: external, middle and inner ear (Table 1). While external and middle ear disease can be due to associated conditions, the most common ear manifestation of IBD is in the inner ear, largely as a result of AIED, and especially in UC. This can be managed effectively through immunosuppression, initially through corticosteroids and stepping up to anti-TNF therapy,
Table 1. Ear Manifestations in IBD

\begin{tabular}{ll}
\hline Site & Diseases \\
\hline External Ear & Metastatic Crohn's disease \\
& $\begin{array}{l}\text { Pyoderma gangrenosum } \\
\text { Relapsing polychondritis } \\
\text { Necrotizing external otitis }\end{array}$ \\
Middle Ear & Otitis media \\
Inner Ear & Sensorineural hearing loss \\
& Autoimmune inner ear disease \\
& Cogan's syndrome \\
& Melkersson-Rosenthal syndrome \\
& Drug-induced ototoxicity \\
\hline
\end{tabular}

and if that fails, cochlear implantation. There are also other associated conditions that may affect the inner ear in IBD patients, such as Cogan's syndrome and Melkersson-Rosenthal syndrome, but these are very rare. In addition, while there is still no evidence to suggest that IBD treatment is associated with ear disease, this requires further investigation as the treatments are recognized to be ototoxic.

\section{References}

1. Barna BP, Hughes GB. Autoimmunity and otologic disease: clinical and experimental aspects. Clin Lab Med. 1988;8(2):385-398.

2. Powell FC, Schroeter AL, Su WP, Perry HO. Pyoderma gangrenosum: a review of 86 patients. Q J Med. 1985;55(217):173-186.

3. Langan SM, Groves RW, Card TR, Gulliford MC. Incidence, mortality, and disease associations of pyoderma gangrenosum in the United Kingdom: a retrospective cohort study. J Invest Dermatol. 2012;132(9):2166-2170.

4. Ben Chaabane N, Hellara O, Ben Mansour W, Ben Mansour I, Melki W, Loghmeri H, Safer L, et al. Auricular pyoderma gangrenosum associated with Crohn's disease. Tunis Med. 2012;90(5):414-415.

5. Lysy J, Zimmerman J, Ackerman Z, Reifen E. Atypical auricular pyoderma gangrenosum simulating fungal infection. J Clin Gastroenterol. 1989;11(5):561-564.

6. Agarwal A, Andrews JM. Systematic review: IBDassociated pyoderma gangrenosum in the biologic era, the response to therapy. Aliment Pharmacol Ther. 2013;38(6):563-572.

7. Arguelles-Arias F, Castro-Laria L, Lobaton T, AguasPeris M, Rojas-Feria M, Barreiro-de Acosta M, SotoEscribano P, et al. Characteristics and treatment of pyoderma gangrenosum in inflammatory bowel disease. Dig Dis Sci. 2013;58(10):2949-2954.

8. Shelley ED, Shelley WB. Cyclosporine therapy for pyoderma gangrenosum associated with sclerosing cholangitis and ulcerative colitis. J Am Acad Dermatol. 1988;18(5 Pt 1):1084-1088. 
9. Chuah JH, Kim DS, Allen C, Hollis L. Metastatic Crohn's disease of the ear. Int J Otolaryngol. 2009;2009:871567.

10. McCallum DI, Gray WM. Metastatic Crohn's disease. Br J Dermatol. 1976;95(5):551-554.

11. Raynor EM. Chronic granulomatous otitis externa as an initial presentation of cutaneous Crohn disease. JAMA Otolaryngol Head Neck Surg. 2014;140(8):765-767.

12. Arnaud L, Mathian A, Haroche J, Gorochov G, Amoura Z. Pathogenesis of relapsing polychondritis: a 2013 update. Autoimmun Rev. 2014;13(2):90-95.

13. Cantarini L, Vitale A, Brizi MG, Caso F, Frediani B, Punzi L, Galeazzi M, et al. Diagnosis and classification of relapsing polychondritis. J Autoimmun. 2014;48-49:53-59.

14. McAdam LP, O'Hanlan MA, Bluestone R, Pearson CM. Relapsing polychondritis: prospective study of 23 patients and a review of the literature. Medicine (Baltimore). 1976;55(3):193-215.

15. Touma DJ, Gross EJ, Karmody CS, Fawaz KA. Relapsing polychondritis in association with Crohn's disease. Am J Otolaryngol. 1996;17(6):424-426.

16. Asuncion AM, Federman DG, Kirsner RS. Swelling of the ear in a patient with ulcerative colitis. Arthritis Rheum. 1994;37(3):432-434.

17. Karmacharya P, Pathak R, Shrestha P, Alweis R. The red hearing: swollen ear in a patient with ulcerative colitis. J Community Hosp Intern Med Perspect. 2014;4(3):24659.

18. Duque G, Portela F, Migueis MC. Necrotizing external otitis on a Crohn's disease patient treated with infliximab. J Crohns Colitis. 2010;4(2):215-216.

19. Shaw SY, Blanchard JF, Bernstein CN. Association between early childhood otitis media and pediatric inflammatory bowel disease: an exploratory population-based analysis. J Pediatr. 2013;162(3):510-514.

20. Shaw SY, Blanchard JF, Bernstein CN. Association between the use of antibiotics and new diagnoses of Crohn's disease and ulcerative colitis. Am J Gastroenterol. 2011;106(12):2133-2142.

21. Karmody CS, Valdez TA, Desai U, Blevins NH. Sensorineural hearing loss in patients with inflammatory bowel disease. Am J Otolaryngol. 2009;30(3):166-170.

22. Akbayir N, Calis AB, Alkim C, Sokmen HM, Erdem L, Ozbal A, Bolukbas F, et al. Sensorineural hearing loss in patients with inflammatory bowel disease: a subclinical extraintestinal manifestation. Dig Dis Sci. 2005;50(10):1938-1945.

23. Kalyoncu D, Urganci N, Calis AB, Ozbal A. Sensorineural hearing loss in pediatric patients with inflammatory bowel disease. Dig Dis Sci. 2010;55(1):150-152.

24. Lobo DR, Garcia-Berrocal JR, Ramirez-Camacho R. New prospects in the diagnosis and treatment of immune-mediated inner ear disease. World J Methodol. 2014;4(2):91-98.

25. Harris JP, Sharp PA. Inner ear autoantibodies in patients with rapidly progressive sensorineural hearing loss. Laryngoscope. 1990;100(5):516-524.

26. Moscicki RA, San Martin JE, Quintero CH, Rauch SD, Nadol JB, Jr., Bloch KJ. Serum antibody to inner ear proteins in patients with progressive hearing loss. Correlation with disease activity and response to corticosteroid treatment. JAMA. 1994;272(8):611-616.

27. Garcia Berrocal JR, Ramirez-Camacho R, Arellano B, Vargas JA. Validity of the Western blot immunoassay for heat shock protein-70 in associated and isolated immunorelated inner ear disease. Laryngoscope. 2002;112(2):304-309.

28. Yeom K, Gray J, Nair TS, Arts HA, Telian SA, Disher MJ, El-Kashlan H, et al. Antibodies to HSP-70 in normal donors and autoimmune hearing loss patients. Laryngoscope. 2003;113(10):1770-1776.

29. Vambutas A, Pathak S. AAO: Autoimmune and Autoinflammatory (Disease) in Otology: what is new in immune-mediated hearing loss. Laryngoscope Investig Otolaryngol. 2016;1(5):110-115.

30. Kariya S, Fukushima K, Kataoka Y, Tominaga S, Nishizaki K. Inner-ear obliteration in ulcerative colitis patients with sensorineural hearing loss. J Laryngol Otol. 2008;122(8):871-874.

31. Broughton SS, Meyerhoff WE, Cohen SB. Immune-mediated inner ear disease: 10-year experience. Semin Arthritis Rheum. 2004;34(2):544-548.

32. Svrakic M, Pathak S, Goldofsky E, Hoffman R, Chandrasekhar SS, Sperling N, Alexiades G, et al. Diagnostic and prognostic utility of measuring tumor necrosis factor in the peripheral circulation of patients with immune-mediated sensorineural hearing loss. Arch Otolaryngol Head Neck Surg. 2012;138(11):1052-1058.

33. Vambutas A, Lesser M, Mullooly V, Pathak S, Zahtz G, Rosen L, Goldofsky E. Early efficacy trial of anakinra in corticosteroid-resistant autoimmune inner ear disease. J Clin Invest. 2014;124(9):4115-4122.

34. Liu YC, Rubin R, Sataloff RT. Treatment-refractory autoimmune sensorineural hearing loss: response to infliximab. Ear Nose Throat J. 2011;90(1):23-28.

35. Heywood RL, Hadavi S, Donnelly S, Patel N. Infliximab for autoimmune inner ear disease: case report and literature review. J Laryngol Otol. 2013;127(11):1145-1147.

36. Staecker H, Lefebvre PP. Autoimmune sensorineural hearing loss improved by tumor necrosis factor-alpha blockade: a case report. Acta Otolaryngol. 2002;122(6):684687.

37. Weisert JU, Veraguth D, Probst R. [Bilateral deafness due to labyrinthitis in a patient with Crohn's disease]. HNO. 2012;60(2):132-134.

38. Kumar BN, Walsh RM, Wilson PS, Carlin WV. Sensorineural hearing loss and ulcerative colitis. J Laryngol Otol. 1997;111(3):277-278.

39. Kuczkowski J, Kozlowski J, Narozny W. [Acute autoimmune sensorineural hearing loss in pregnant women with Lesniowski-Crohn disease]. Otolaryngol Pol. 2006;60(4):583-585.

40. Narbutt P, Dziki A. Re: Melkersson-Rosenthal syndrome as an early manifestation of Crohn's disease. Colorectal Dis. 2005;7(4):420-421.

41. Ilnyckyj A, Aldor TA, Warrington R, Bernstein CN. Crohn's disease and the Melkersson-Rosenthal syndrome. Can J Gastroenterol. 1999;13(2):152-154.

42. Vavricka SR, Greuter T, Scharl M, Mantzaris G, Shitrit AB, Filip R, Karmiris K, et al. Cogan's syndrome in pa- 
tients with inflammatory bowel disease - a case series. J Crohns Colitis. 2015;9(10):886-890.

43. Tayer-Shifman OE, Ilan O, Tovi H, Tal Y. Cogan's syndrome - clinical guidelines and novel therapeutic approaches. Clin Rev Allergy Immunol. 2014;47(1):65-72.

44. Cuffel C, Guyot JP. [Aspirin ototoxicity: a rare situation today]. Rev Med Suisse. 2013;9(400):1785-1788.

45. Iqbal SM, Murthy JG, Banerjee PK, Vishwanathan KA. Metronidazole ototoxicity - report of two cases. J Laryngol Otol. 1999;113(4):355-357.

46. Paul J, Brown NM. Tinnitus and ciprofloxacin. BMJ. 1995;311(6999):232. 